\title{
Improving Management of Osteoarthritis: Patients' Perceptions of a Surgical Readiness Interview Tool
}

\author{
Barton $\mathrm{KI}^{1,2,3 *}$, Hewison $\mathrm{CE}^{4}$ and Kania-Richmond \\ $\mathbf{A}^{5,6}$ \\ ${ }^{1}$ Schulich School of Dentistry and Medicine, Western \\ University, London, ON, Canada \\ ${ }^{2}$ School of Physical Therapy, Faculty of Health Sciences, \\ Western University, London, ON, Canada \\ ${ }^{3}$ Faculty of Kinesiology, University of Calgary, Calgary, \\ $\mathrm{AB}$, Canada \\ ${ }^{4}$ Cumming School of Medicine, University of Calgary, \\ Calgary, AB, Canada \\ ${ }^{5}$ Bone and J oint Health Strategic Clinical Network, \\ Alberta Health Services, Calgary, AB, Canada \\ ${ }^{6}$ Department of Community Health Sciences, University \\ of Calgary, Calgary, AB, Canada \\ *Corresponding author: Barton KI, Orthopaedic \\ Surgery, Schulich School of Dentistry and Medicine, \\ Western University, 1151 Richmond St, London, ON N6A \\ 5C1, Canada
}

Received: July 14, 2021; Accepted: August 31, 2021; Published: September 07, 2021

\begin{abstract}
Almost half of patients referred to orthopedic surgeons for Total Joint Arthroplasty (TJA) do not require TJA at that time or are not appropriate surgical candidates. The objective was to explore patients' perspectives of a Surgical Readiness Interview Tool and its potential utility in the Osteoarthritis (OA) management process. Semi-structured qualitative interviews were conducted with a convenience sample of patients from arthroplasty clinics in Alberta. All interviews were digitally recorded and transcribed verbatim. Analysis was conducted iteratively, applying a constant comparison method. Nine patients were interviewed. Participants found the interview tool to be relevant and comprehensible. Suggestions were made on how to improve tool clarity and administration processes. Patient orientation versus that of the doctor, and expectation management emerged as salient factors in the meaningful application of the tool. As a result of the interviews, a revised tool was developed which incorporated the participant suggestions. Patients were positive about the interview tool and felt that it would lead to better care provision, particularly with incorporation of participants' suggestions. The data suggest that the interview tool could improve the conversation on surgical readiness, conservative management, and addressing modifiable risk factors prior to TJA.
\end{abstract}

Keywords: Osteoarthritis; Readiness; Arthroplasty; Motivational interviewing; Modifiable risk factors

\section{Abbreviations}

BJH SCN: Bone and Joint Health Strategic Clinical Network; OA: Osteoarthritis; TJA: Total Joint Arthroplasty

\section{Introduction}

Total Joint Arthroplasty (TJA) surgery is an effective and costeffective intervention for severe hip and knee Osteoarthritis (OA) leading to reduce pain, improve function, and improve quality of life [1-8]. In current practice, surgical suitability and medically determined readiness for TJA includes the following factors: degree of OA (progression of OA disease on plain radiographs), level of severity of functional disability or dysfunction, pain experience, patient symptoms, and/or co-morbidities, most notably obesity [9]. However, utilization rates vary, as there are no set indications for when it is best to operate, and which patients benefit most from the operation.

The majority of patients that are referred to orthopedic surgeons for hip and knee OA in Alberta do not require TJA at that time or are not appropriate surgical candidates. McHugh et al. report that patient referrals to an orthopedic surgeon by general practitioners were often inappropriate, with only $33 \%$ of referred patients undergoing TJA [10]. Of the remaining patients, the majority were sent back to their family physician because they did not want to undergo TJA or they had not yet exhausted non-surgical options (i.e. physiotherapy, joint injections, weight loss), had been referred to the wrong type of specialist (i.e. referred for arthroplasty when should have been referred high tibial osteotomy), or were not in a condition severe enough to warrant TJA [10]. Canadian data suggests that $45 \%$ of patients with moderate knee OA who are referred by their family physician to an orthopaedic surgeons do not require TJA at that time or are not appropriate surgical candidates [11,12]. Klett et al. [13] also found that $47.4 \%$ of patients referred to sports medicine specialists were referred back to their primary care physician. Furthermore, patients referred to TJA by a sport medicine specialist were more likely to have exhausted conservative measures to manage their OA [13], utilizing conservative management prior to TJA. Similarly, in a study by Cross et al. [14], orthopaedic surgeons were found to be focused on disease management (disease severity, obesity, severity, patient demand, nursing home residency, etc.) in comparison to referring health care providers, which suggests the importance surgeons place on exhausting conservative management before proceeding with TJA.

One proposed solution to address this important point was the development of a Surgical Readiness Interview Tool (interview tool) that would facilitate a conversation between a family physician and a patient about modifiable risk factors and non-surgical management strategies for OA. Further, there was no other screening tool used being used by family physicians prior to a referral for an orthopedic consult to evaluate readiness for TJA and to identify modifiable risk factors that may contribute to adverse surgical outcomes. Lastly, it was recognized that such an interview tool might also enable a supportive dialogue with a shift to emphasize non-surgical approaches, which would be more beneficial to the patients' overall health.

The interview tool was developed by assessing the current evidence-based guidelines and recommendations for both TJA and 
optimizing surgical outcomes (Table 1). The language used was based on current Motivational Interviewing (MI) techniques and used to identify patient knowledge, engagement, and ability to change [15]. The interview tool also included evidence-based non-surgical management strategies to ensure patients are aware of and have exhausted all other treatment options prior to a consult for surgery.

The interview tool has been presented to clinicians and clinical support staff of arthroplasty clinics from across Alberta at provincial meetings of the Bone and Joint Health Strategic Clinical Network (BJH SCN), in particular a BJH SCN workshop focused on care for individuals living with $\mathrm{OA}$ and obesity [16]. Although there was interest from clinics to test the tool in their settings, it was recognized that a key stakeholder in the uptake and utility of this tool are patients. Hence, input from patients to confirm utility and alignment with patient needs was obtained. Further, with patient input, there was need to determine the optimal operational placement of the tool. For example, primary care settings or central intake hip and knee clinics. The objective of the qualitative inquiry was to explore patients' perspectives of the interview tool and its potential utility in the management process for those with $\mathrm{OA}$, with the goal of improving conversation with regard to surgical readiness and management of modifiable risk factors.

\section{Methods}

Ethical approval was received from the Conjoint Research Ethics Board at the University of Calgary (Ethics ID: REB17-1814). All participants provided verbal informed consent prior to the telephone interview being conducted. A qualitative study was conducted and COREQ guidelines for qualitative research reporting were followed [17]. Interpretative description was used as it enables an in-depth exploration of a participant's experience and perceptions and moves beyond description by intentionally aligning the lived experience and implications within the clinical or health care.

The study was conducted in Calgary, Alberta, Canada with patients recruited from two hip and knee arthroplasty clinics, who had previously undergone at least one TJA at one of the recruitment sites. A convenience sampling strategy was used with the aim of including a range of participants (male/female; broad age range; no requirements related to education, residence location or other underlying conditions). The final sample was informed by data saturation. Recruitment was stopped when it was determined that no new codes or categories would emerge with additional interviews. Potential participants were approached about the study by clinic managers during their routine post-TJA follow up appointment. If consent to be contacted by the research team was obtained, contact for the interviews was initiated by a research team member, with study consent obtained verbally prior to the interview.

Semi-structured qualitative interviews were conducted by telephone by KIB, NLT and CEH. The interviews applied a cognitive interviewing approach in order to elicit information regarding clarity of questions and response options, relevance of topics covered in the tool, and what may be missing in the tool when considering readiness for arthroplasty. Based on a preliminary analysis of the first two (2) interviews, AKR provided minor revisions to the interview guide and additional coaching on how to frame questions in a semi-structured format during the interview process. All interviews were digitally recorded and transcribed verbatim.

The analysis was initiated after the first 3 interviews were completed. Each transcript was independently analyzed by two team members (AKR, KIB). Codes and categories identified by each member were compared to determine corroboration through three rounds. Any discrepancies were further discussed and final decisions regarding codes/categories were consensus-based. Through several iterations, an emergent coding framework was developed, and applied to all interviews for descriptive and thematic (interpretative) coding (Table 3).

\section{Results}

The participant sample $(n=9)$ included males $(n=3)$ and females $(\mathrm{n}=6)$, and, at the time of the interview, all had at least one TJA within the previous 12 months. Overall, participants were positive about the tool and felt that it would lead to better care provision. They felt the questions were clear and relevant to determining surgical readiness for individuals with osteoarthritis, and adequate for addressing modifiable risk factors, as well as addressing issues relevant to arthroplasty. The rating scale used in the interview tool to assess each item was seen as effective in capturing responses as it provided a quick yet good sense of where participants were at in relation to each of the health areas addressed.

There appeared be nothing surprising in the topics covered in the interview tool. For some, there was a degree of familiarity with the content of the tool; several participants $(n=6)$ said that these types of questions were addressed with their family doctor or physiotherapist in relation to their OA and/or discussions regarding surgery. Several participants $(n=7)$ also commented that it was a good source of credible information in terms of what needs should be considered in relation to surgery. Some participants $(n=3)$ also emphasized that this information is not only useful for them, but also for their family doctors and surgeons as it provided a more complete picture of what the patient had done (or not). All expressed that they felt comfortable discussing the content with their doctors.

The key areas that raised questions for participants were related to tool administration, question priority, and relative importance of the topics in relation to determining readiness for surgery. A few of the participants were unclear about the method of administration whether it would be done by the patient alone or with a doctor, or by the doctor. Timing of administration, in relation to a referral or scheduling of a surgery, was also raised by a few participants. This was important as it appeared to determine the purpose of the tool: later administration seemed to mean it would be used as a decisionmaking tool for the doctor regarding referral to a specialist for surgery whereas earlier administration meant it functioned more as tool for the patient to help assess what needed to be done to enhance their surgical readiness. There was some preference towards earlier administration and using the tool as a readiness tool for the patient.

There was some question as to whether the order of questions reflected a priority in relation to surgical readiness, which suggested that the first topics were more important than those listed towards the end. Related to this, some participants were unclear about the relative importance of the questions. For example, almost all agreed that exercise and physical strength were important, however, 
Table 1: The readiness interview tool for patients to improve conversation on modifiable risk factors prior to total joint arthroplasty (TJA). The revised suggested changes are in grey text.

\section{Strength and Physical Fitness}

Do you feel your strength and overall fitness will impact your hip or knee osteoarthritis?

How important is it to you to change your strength and overall fitness?

How confident are you that you can change your strength and overall fitness?

\section{Eating Habits}

Do you feel your eating habits will impact your hip or knee osteoarthritis?

How important is it to you to change your eating habits?

How confident are you that you can change your eating habits?

\section{Smoking / Tobacco Use}

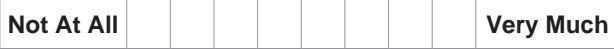

Do you currently smoke or chew tobacco? $\square$ Yes $\square$ No $\rightarrow$ Skip to Section 4

Do you feel your smoking will impact your hip or knee osteoarthritis?

How important is it to you to change your smoking habits?

How confident are you that you can change your smoking habits?

\section{Body Weight}

Do you feel as though you have excess weight?

Do you feel your weight is well controlled and/or maintained?

Do you feel your weight will impact your hip or knee osteoarthritis?

How important is it to you to change your weight (if applicable)?

How confident are you that you can change your weight (if applicable)?

\section{Diabetes}

Do you have diabetes (Type 1 or Type 2)? $\square$ Yes $\square$ No $\rightarrow$ Skip to Section 6

Do you feel your diabetes is well controlled?

Do you feel your diabetes will impact your hip or knee osteoarthritis?

How important is it to you to change your diabetes management?

How confident are you that you can change your diabetes management?

6. Check All Strategies That You Have Tried (please provide any details about if these worked and

to what extent)

Physiotherapy (please list):

Exercise Therapy (please list):

Braces (please list):

Joint Injections (e.g. corticosteroids (cortisone), hyaluronic acid (Synvisc, Durolane), plasma rich protein, stem cell therapy; please list type/timing):

Medications Specific to Joint Problems and Pain Management (e.g. anti-inflammatories, chondroitin sulfate, glucosamine, over the counter drugs, narcotics,

painkillers, topicals (Voltaren); please list):

Other (e.g. walking aids, orthotics please list)

\section{Readiness for Surgery}

To what extent is your hip or knee osteoarthritis impacting your daily life?

To what extent is your hip or knee osteoarthritis limiting your daily activities?

To what extent is your hip or knee osteoarthritis impacting your mental health?

Do you believe that you understand what the surgery entails?

Do you believe that you understand what the surgery rehabilitation entails?

How ready are you to undergo surgery?

Do you feel surgery will benefit you?

questions were raised regarding the relevance of the weight question. This was due to the inconsistency observed, as discussed by several participants, regarding excess weight and its impact on surgery and/ or surgical outcomes. For example, several participants observed that some people who were over-weight had surgery "... and did just fine". Some who were not over-weight had poor outcomes. Therefore, focus 
Table 2: Suggested changes for the readiness interview tool from study participants.

\section{Terminology}

Term "Excess Weight"

Participant 1: “...and your concern about just the words excess weight. Right? Okay. Um, I, I really don't know any other way to put it myself. Other than maybe you can call it weight management or something like that."

Participant 2: "...like other than maybe changing the excess weight title and I don't know what you would change it to-um, I think other than that, it looks good."

Participant 3: "...I think that's what, that's the big thing I would just change that to just either weight or body weight, something like that."

Participant 3: "I think just maybe just calling it, you know, body weight, or weight might seem, might make you feel less defensive about it."

Participant 3: “...the only thing that bothered me was in [question] number four uh where it's the excess weight. So, the weight thing isn't bothersome. I think the use of the word excess. Um, I think you're sort of assuming perhaps, that people are overweight? Who has these issues? And I certainly have known people who were not. So, I think if it was just perhaps renamed weight. Or body weight. Um, I think just, there's an inference here that you must be overweight, right. So that the word excess is just kind of, it already put, kind of puts you in a bit of a defensive mode."

Participant 8:“...the nurses take you and uh do your height and your weight and stuff like this. And he came in with this piece of paper, and I guess it had my weight on there as well as my height and stuff like this. And he says, you're obese. And I said, what?! I said, what the heck are you talking about? I said, yes, I've put on a few pounds. I said, I may be 10 to 15 pounds overweight, but you call that obese? I said, what is wrong with you? I, I just wanted to up and hammer him right there, I was so angry with him". And the word obese should never be used to a person that is trying to stay fit with a problem knee. You know?" Implied Assumption (that the person is overweight in question 4)

Participant 3: "...the only thing that bothered me was in [question] number four uh where it's the excess weight So, the weight thing isn't bothersome. I think the use of the word excess. Um, I think you're sort of assuming perhaps, that people are overweight? Who has these issues? And I certainly have known people who were not. So, I think if it was just perhaps renamed weight. Or body weight. Um, I think just, there's an inference here that you must be overweight, right? So that the word excess is just kind of, it already put, kind of puts you in a bit of a defensive mode.

Participant 7: "I had some difficulty answering some of the questions-only some of them are very straightforward. But I, I thought some of the questions were somewhat assuming some things, depending on what your answer was."

Confidence

Participant 6: "And then how confident are you? For some reason, it just doesn't resonate with me when it's, when it's stated that way."

Spacing

Ability to Include Details (provide a better/more complete/more comprehensive picture to the physician)

Participant 2: "Some doctors are pretty good at, you know, trying to get you to try other things. Others like, l'm looking especially at joint injections."

Participant 2: "...like and even the weight thing. Like I said, I know this guy was deflated and he'd tried losing weight, so I don't know what you-it's a difficult one, right? And people are sensitive."

Participant 3: "The only thing I can think of was, just as an example, like for [question] number seven joint Injections, l've also had Synvisc. So, it just wasn't listed there. I know that a lot of people have had that."

Participant 4: "There's not enough space, like if you've tried exercise therapy and physiotherapy and the braces and you're supposed to list all of it, hopefully there's room, you know, some place where you can add all that stuff. Because you know, if you've done the last check off strategies that you have tried, but then it says, please list, so then you start listing all the things that you've tried, you kind of run out of space on that sheet."

Include Additional Information

Additional Information Discussed (mental health measure/indicator, quality of life, blood pressure, impact on everyday life, rating of activity, impact of joint disease on function)

Participant 1: "Um, the only other thing is, is do you, do we, do you need to put in things like high blood pressure?"

Participant 3: "The one thing about-like for me personally, I have severe OA in both knees. And I have two small children. Is how this impacts daily life? So, you know, you, you're going over things like strength and your eating and your smoking and your weight. But perhaps, like trying to gauge like people's pain level. Um and, and the kind of impact-like that is maybe where the conversation would come in. Like, how much of this really is affecting your life? Um, I know for me it affects me on every level. Um everything I do. So um, that might help a surgeon or a doctor gauge, like how important the surgery is. I mean, of course, there is always the criteria of you know, is this going to be helpful? Is this something that you know, you can actually do? But the fact that it does impact people's lives so much. What is having OA like, how does it, affect your daily life? And what is your pain level? How do you tolerate that? And how do you get through life on a daily basis like that? Might help them, you know."

Participant 7: Recommended rating of activity, quality of life, and impact of joint disease on function

Participant 8: "People that are not uh well, um physically are not well mentally either, you know. And u, you know, l'm not saying a shrink or anything like that, but you know, it, um, it has a tendency to play mind games on the, on the, on-on the head. So maybe incorporating a mental health question would be beneficial too for the Tool?

Participant 8: Recommended listing topical creams for question 7.

\section{Readiness}

Ready at Home Post-Surgery/Discharge

Participant 3: "Yeah so like for instance, for me, I had an ACL reconstruction. And I was a single girl at the time. And living by myself. And so um, you really, you have to organize yourself. I went and I got a whole bunch of um food that could readily be made. Um I was on crutches. So, I had to do things like um, put yeah easily, easily prepared food in a plastic bag and put it, and hold it in a plastic bag with my crutches, and go over to the table. Right? So even, you know, and like having a tea was, I couldn't. I couldn't make tea, because I couldn't carry it unless I stood there and drank the tea. So, it's all these little things that um, your after care. Like do you, is it, is it possible for you to go through this surgery? And be able to look after yourself? Um afterwards. Um it is quite important. That's something. It took me two and a half months to recover from that surgery. And be able to go back to work in a standing position. And so it's a long time and everything from like bathing, you know. Like do you have help? You know, is there somebody who can come in? Do you have help? Are you aware? And I didn't know this at the time-that there is Home Care. Um there's all these types of things. And what, what um, is accessible to you? So, it's a big part of going through this type of surgery. At least it was for me."

Participant 3: "Like if you added a couple extra spots, you know? Like, like I say, for things like um, like how does this impact your daily life? And the pain level. And then things like after care, like are you prepared to be virtually incapacitated, to some degree? And, or like, can you get yourself to um physio? You know? I had to recruit my brother-in-law to drive me to Physio over by what used to be called the Talisman."

Readiness for Surgery (both going into surgery and coming out of surgery)

Participant 3: “... people might not think about um, your strength and your physicality. Like, l've had three knee surgeries. And so, I know that if you have a, a very weak musculature, that after surgery your road back is a lot harder. So, people might not realize that. They might think it's, you know, going in for an, an appendix uh taken out, and you can just keep going. Like the, the more in shape you are, and um the better your muscles are toned, you will, it'll help your recovery." Participant 3: "But when it really comes to the topic of surgery, it's not just are you physically capable to go into surgery? But how are you going to be coming out?" Participant 4: "...and because like, you get these exercises, like you can go online and you can go to the Hip and Knee Clinic and they show you these exercises, and yeah they're all a piece of cake before surgery. You know? Like these things, all are a piece of cake until you really, until you have had the surgery. And then it's a whole different ballpark." 


Administration
Timing (complete earlier rather than later)
Participant 5:
"I guess it depends on how far in advance you are, um, um, the doctor's going over this Tool with you because if your surgery is you know, six months away or four
months away or whatever, there's um, in, I don't know how much you can do in terms of losing weight if that's something that um, um, you need to consider. Or, or
getting, strengthening, I think the, the earlier on in the process this is, this tool is used, the better."

Table 3: Emergent coding framework.

\begin{tabular}{|c|c|c|}
\hline Category & Sub-Category & Code \\
\hline \multirow{14}{*}{ Positive Response } & \multirow{3}{*}{ Good source information } & Knowing factors that impact readiness for surgery (i.e. information, awareness, education tool) \\
\hline & & Credible source of information \\
\hline & & Information for surgeon (what you are implementing and what you have tried) \\
\hline & \multirow{5}{*}{ Appropriate } & Better care provision \\
\hline & & Reasonable questions to answer if surgery is being considered \\
\hline & & The term 'excess weight'; good middle ground, no concern with term \\
\hline & & To discuss with doctor \\
\hline & & For patient with arthritis \\
\hline & \multirow[b]{2}{*}{ Familiarity with topics } & Nothing surprising in the tool \\
\hline & & $\begin{array}{l}\text { Topics not new to participants; asked these types of questions (with doctor or physio) prior to } \\
\text { surgery }\end{array}$ \\
\hline & \multirow{4}{*}{ Structure } & Understandable, clear, and concise \\
\hline & & Good layout \\
\hline & & Like the rating scale \\
\hline & & Strategies tried \\
\hline \multirow{7}{*}{ Clarifications } & \multirow{4}{*}{ Tool administration } & Method for administration (questionnaire vs. conversation guide) \\
\hline & & Clarify the purpose of the tool (clinical tool, guide a conversation, self-check tool for the patient?) \\
\hline & & Method to elicit honest response \\
\hline & & Method of timing (completed earlier rather than later) \\
\hline & Question priority & \\
\hline & \multirow{2}{*}{ Relative importance of topics } & Contradictory information about the importance of weight/weight loss for surgical readiness \\
\hline & & Connection between topics \\
\hline \multirow{3}{*}{ Managing Expectations } & Risk assessment & What if the surgery is not successful? \\
\hline & Change expectations & Surgeon requires weight loss, exercise, further management \\
\hline & Expectation of a 'truthful' response & \\
\hline
\end{tabular}

on or any emphasis on weight seemed contradictory or confusing to participants. However, through the analytic process, two themes emerged-patient orientation and expectation management-which are described below.

Several changes to the language and content were proposed by participants to further enhance the tool and enable its orientation towards the patient and their needs. In terms of terminology used in the questions, asking about a patient's confidence in relation to their readiness did not "resonate" for one participant, as they did not think this was an issue of confidence. Several participants were dissatisfied with the question specific to weight management. The wording seemed to imply that weight was a concern or an issue, which "automatically makes one defensive ..." (Participant 3). Similarly, use of "excess weight" was interpreted as an assumption that there was a problem with weight. Whereas stating, "weight' or 'weight management' in the question was perceived to be more neutral, not problematizing weight. Most participants shared the opinion that the use of "excess weight" was preferable to "over-weight" or "obese" or "fat" as these appeared be interpreted as an insult or negative judgement of the person. The sensitized meaning of these words appeared to be important to participants, as reflected in the experience of Participant 8:

"And he (surgeon) came in with this piece of paper... and he says, you're obese. And I said, what?! I said, what the heck are you talking about? I said, yes, I've put on a few pounds. I said, I may be 10 to 15 pounds overweight, but you call that obese? I said, what is wrong with you? I just wanted to up and hammer him right there, I was so angry with him...the word obese should never be used to a person that is trying to stay fit with a problem knee". 
Participants also commented that a narrow view of weight - a number on a scale - had negative consequences. It resulted in an automatic judgement and precluded a beneficial health-focused dialogue. The experience of some participants suggests that this could lead to detrimental effects on a patient's physical/or and mental health, as evidenced in the experience of Participant 8:

"...wording is definitely everything. It can turn their mind off surgery, and they go into, they turn into themselves. And they uh build a shell around them and they don't come out. You know? It takes a long time to reach a person like this".

Participants' feedback identified additional information that should be included in the interview tool: an indicator of mental health state, quality of life and rating of impact of the disease on everyday activities and functionality. A number of participants recommended provision of more space for each question where additional details could be included to provide a better or more complete picture of the individual's reality or situation. Additionally, the different strategies can be listed and the individual's comments specific to the strategies tried. This seemed to be particularly important in relation to exercise and weight management. For instance, simply being labelled as "over-weight" or "obese" without a context or understanding of why was very limiting and did not provide the complete picture that participants felt should be considered by a doctor.

There were several recommendations to expand what is covered under "surgical readiness". Several noted that this does not only relate to the time prior to surgery but also after surgery. To that end, suggestions were made that the tool should include questions that consider readiness at home - food preparation, social and health care supports, access within the home. Lastly, consideration of the very real challenges to be faced after surgery - pain, limited mobility, poor sleep - and if the patient is ready for this. As one participant commented:

“... and they show you these exercises, and yeah they are a piece of cake before surgery... until you've had the surgery. Then it's a whole different ballpark..." (Participant 4).

Participants' input and comments on the tool brought to light the importance of managing expectations of patients and doctors as to what the tool outcomes mean and their relevance in terms of healthcare provision. Several participants raised the issue that any discussion about surgery, even if the tool suggests a high readiness level, should also include some real risk considerations - the surgery may not work, the outcomes may be poor. Further, the level of functionality may improve but it will not be at the same as level as before they had OA. As one participant stated:

“....I knew, once I had the procedure done I wasn't going to be ... it's not as good as my original equipment... and I did not expect it to be....as a result, I'm definitely not disappointed" (Participant 9).

The interview tool may also be useful in potentially managing the expectations that doctors have of their patients in terms of what can be realistically achieved by the person compared to a readiness and/ or optimization benchmark. Although most participants recognized the importance health factors listed in the tool, many expressed a lack of control of the issues their doctors wanted addressed or "fixed". For example:
“... and I think sometimes people, especially if they have no issues with weight, always just say well you've just got to lose it, you've got to lose weight. But this guy was really trying. And he gets to meet the surgeon finally - and it takes a long time to get to that point - and I remember he was just so deflated. And he just felt like, he didn't know what the next step was" (Participant 2).

Some also spoke of a 'vicious cycle' that doctors appeared unaware of, and, in some ways, was perpetuated by doctors. For instance, one became overweight because they were unable to exercise due to the arthritis and the pain. And now they were expected to lose weight that they gained because they are unable to function and did not have surgery that they perceived would give them the function back.

Lastly, there is an expectation that people will respond to the questions in the tool honestly. However, one participant pointed out that if the tool is used to inform a decision about surgery or referral to a surgeon, this may influence how the patient responds to the questions; they may aim to provide the "right answer" instead of an honest answer to get the end result they want - the surgical referral and/or the surgery.

\section{Discussion}

The intent of this interview tool was to involve the patient and enable better communication between the doctor and the patient regarding management of the patient's OA; therefore, focusing on a patient centric care to align decisions with patient's needs, wants, and preferences [18-20]. Overall, participants were positive about the interview tool and felt that it would lead to better care provision. However, a common perspective identified across multiple interviews was that the tool was actually more medicine or physician centric. Although study participants recognized it to be a useful educational and awareness tool in OA management, it appeared to be perceived as primarily oriented towards information needs and issues of importance to physicians and not necessarily the patient. To ensure that the tool is also meaningful to patients in the context of their lives and decision-making regarding surgery, input from study participants was incorporated in the interview tool. The revised tool is provided in Table 2 .

An important issue that emerged through the analytic process was related to how patients respond to questions. The tool creates the opportunity for an open dialogue that may help identify which risk factors the patient is willing and able to start making changes on. This often comes with an inherent assumption, if not expectation, that patients respond to questionnaires or questions, in the context of a consultation with a physician, in an honest or transparent manner. However, several participants identified that this is not always the case. Some will frame their response based on what they perceive the physician may want to hear in order to achieve a certain 'next step' in a care process, such as a surgical referral. Therefore, when it's administered and what the results of the tool are used for will likely influence how a patient responds to the questions. A similar issue was addressed in a study by Burt et al (2017) exploring how patients' choices of response options related the nature of the primary care physician consultation. The authors concluded that drivers impacting responses to questionnaires include the nature of the consultation with a primary care physician and expectations of that consultation, and the power differential between a doctor and the patient [21]. This 
highlights the need for careful consideration of when the interview is administered in the process of OA management and establishing clear expectations, as this may potentially influence how a patient responds to the tool items.

Time of the interview tool administration within the care journey of individuals living with $\mathrm{OA}$ was an important factor and appeared to have implications on how the tool could be both used and interpreted. Although initially the tool was presented to orthopedic specialists, participant feedback suggests its utility may be greater within a primary care setting. This aligns with the stepped care approach applied by the BJH SCN framework for osteoarthritis management [22]. The stepped care approach [23] provides a progressive strategy to management and treatment of OA that is initiated with low intensity evidence-informed interventions and an emphasis on self-management in (step 1). This is progressed to increasingly more intensive treatment interventions with step 2, including exercise therapy, dietary therapy, and non-steroidal antiinflammatory drugs, and step 3 , comprising of multidisciplinary care, intra-articular injections, and transcutaneous electrical nerve stimulation for patients with persisting complaints. Surgery would be the last option, after step 3 essentially failed in effective treatment of OA symptoms. With the stepped care approach, it is imperative that the steps are completed in the prescribed manner. Further, to provide value, a stepped care strategy needs to be consistent across the different providers involved (e.g. family physicians, specialists), as there are effects on costs and long-term effects [23]. Within this stepped care approach, the interview tool could provide consistency within the primary care setting as to what conversations to have prior to any referrals, including consultation for arthroplasty, and engage the patient in playing an active role in their care. The interview tool may also prompt the family physician to encourage follow up appointments for both discussion and reassessment of modifiable risk factors, and guide patients to appropriate non-surgical interventions (i.e. physiotherapists, kinesiologists, dieticians, diabetes management clinics, smoking cessation clinics, etc.) prior to a surgical referral. Lastly, its uptake could result in improvements in referral efficiency by appropriately triaging poor current surgical candidates to other health care professionals, which would in turn reduce inappropriate surgeon referrals.

This study is not without limitations. First, the patients were recruited within specific geographic location - Calgary, Alberta; however, these clinics service a larger proportion of hip and knee patients in Alberta and are not limited to Calgary residents. Second, the patients were identified by clinic managers and then invited to participate, therefore introducing potential selection bias into which patients were invited to be interviewed. Lastly, the cognitive interviewing approach was relatively new to the interviewers. This led to some inconsistency in how the interviews were conducted by the three interviewers. However, the semi-structured approach allows for flexibility in the flow of an interview to ensure research objectives are addressed. Further, coaching was provided and almost all interviews (6/9) were conducted by the same interviewer (KB). Peer member checking of the revised tool was not conducted. It may be step incorporated into an implementation strategy to confirm acceptability of the interview tool from the patient perspective.

\section{Conclusion}

In summary, patient participants were positive about the proposed interview tool. Participants believed these discussions would lead to better care. The use of this tool will likely enable better patient engagement in managing chronic diseases and have real impact in reducing the number of inappropriate consults seen by orthopedic surgeons. The results confirmed utility of the interview tool to improve the conversation on surgical readiness, conservative management, and addressing modifiable risk factors that contribute to adverse surgical outcomes in TJA.

\section{Declaration}

Author's contributions: KIB co-developed the tool, implemented revisions to create version 2 of the tool, collaborated on the study design, conducted interviews, analyzed data and led the manuscript preparation. CEH co-developed the tool development, conducted interviews, and edited the manuscript. AKR collaborated on the study design, confirmed version 2 of the tool, data analysis and contributed to the manuscript preparation.

Acknowledgements: The authors would like to acknowledge Dr. Nicole Thompson for assistance in tool development. The authors would also like to acknowledge the study participants for their time and insights.

\section{References}

1. Ethgen $O$, Bruyère $O$, Richy $F$, Dardennes $C$, Reginster JY. Health-related quality of life in total hip and total knee arthroplasty. A qualitative and systematic review of the literature. J Bone Joint Surg Am. 2004; 86-a: 963974.

2. Fortin PR, Clarke AE, Joseph L, Liang MH, Tanzer M, Ferland D, et al. Outcomes of total hip and knee replacement: preoperative functional status predicts outcomes at six months after surgery. Arthritis Rheum. 1999; 42: 1722-1728.

3. Jones CA, Beaupre LA, Johnston DW, Suarez-Almazor ME. Total joint arthroplasties: current concepts of patient outcomes after surgery. Rheum Dis Clin North Am. 2007; 33: 71-86.

4. Judge A, Arden NK, Cooper C, Javaid MK, Carr AJ, Field RE, et al. Predictors of outcomes of total knee replacement surgery. Rheumatology (Oxford). 2012; 51: 1804-1813.

5. Laupacis A, Bourne R, Rorabeck C, Feeny D, Wong C, Tugwell P, et al. The effect of elective total hip replacement on health-related quality of life. J Bone Joint Surg Am. 1993; 75: 1619-1626.

6. Rampersaud YR, Wai EK, Fisher CG, Yee AJM, Dvorak MFS, Finkelstein JA, et al. Postoperative improvement in health-related quality of life: a national comparison of surgical treatment for focal (one- to two-level) lumbar spinal stenosis compared with total joint arthroplasty for osteoarthritis. Spine J. 2011; 11: 1033-1041.

7. Salmon P, Hall GM, Peerbhoy D, Shenkin A, Parker C. Recovery from hip and knee arthroplasty: Patients' perspective on pain, function, quality of life, and well-being up to 6 months postoperatively. Arch Phys Med Rehabil. 2001; 82: 360-366.

8. Dieppe PK, Lim K, Lohmander S. Who should have knee joint replacement surgery for osteoarthritis? Int J Rheum Dis. 2011; 14: 175-180.

9. Frankel L, Sanmartin C, Conner-Spady B, Marshall DA, Freeman-Collins L, Wall A, et al. Osteoarthritis patients' perceptions of "appropriateness" for total joint replacement surgery. Osteoarthritis Cartilage. 2012; 20: 967-973.

10. McHugh GA, Campbell M, Luker KA. GP referral of patients with osteoarthritis for consideration of total joint replacement: a longitudinal study. $\mathrm{Br} \mathrm{J}$ Gen Pract. 2011; 61: e459-468. 
11. Malian SJ. Predictors of appropriate referral to total knee arthroplasty: validation study, in Health and Rehabilitation Sciences. Western University: Electronic Thesis and Dissertation Repository. 2015: 75.

12. Churchill L, Malian SJ, Chesworth BM, Bryant D, MacDonald SJ, Marsh JD, et al. The development and validation of a multivariable model to predict whether patients referred for total knee replacement are suitable surgical candidates at the time of initial consultation. Can J Surg. 2016; 59: 407-414.

13. Klett MJ, Frankovich R, Dervin GF, Stacey D. Impact of a surgical screening clinic for patients with knee osteoarthritis: a descriptive study. Clin J Sport Med. 2012; 22: 274-277.

14. Cross WW, Saleh KJ, Wilt TJ, Kane RL. Agreement about indications for total knee arthroplasty. Clin Orthop Relat Res. 2006; 446: 34-39.

15. Dietz WH, Kahan S. Why weight? A guide to discussing obesity and health with your patients. Strategies to Overcome and Prevent (STOP) Obesity Alliance. 2014.

16. Godziuk K, Kania-Richmond A. Hart D. Obesity: Implications for patients with osteoarthritis. 2017.

17. Tong A, Sainsbury P, Craig J. Consolidated criteria for reporting qualitative research (COREQ): a 32-item checklist for interviews and focus groups. Int J Qual Health Care. 2007; 19: 349-357.

18. Bardes CL. Defining "patient-centered medicine". N Engl J Med. 2012; 366 782-783

19. Barry MJ, Edgman-Levitan S. Shared decision making--pinnacle of patientcentered care. N Engl J Med. 2012; 366: 780-781.

20. Weiner SJ, Schwartz A, Sharma G, Binns-Calvey A, Ashley N, Kelly B, et al. Patient-centered decision making and health care outcomes: an observational study. Ann Intern Med. 2013; 158: 573-579.

21. Thorne S, Kirkham SR, O'Flynn-Magee K. The Analytic Challenge in Interpretive Description. Int J Qual Methods. 2004; 3: 1-11.

22. Bone and Joint Health Strategic Clinical Network. Model of Care for Osteoarthritis Treatment. 2017.

23. Smink AJ, van den Ende CHM, Vlieland TPMV, Bijlsma JWJ, Swierstra BA Kortland $\mathrm{JH}$, et al. Effect of stepped care on health outcomes in patients with osteoarthritis: an observational study in Dutch general practice. $\mathrm{Br}$ J Gen Pract. 2014; 64: e538-544. 